\title{
Consecuencias económicas y sociales de la inamovilidad humana bajo COVID-19: caso de estudio Perú
}

\author{
Israel Barrutia Barreto (10), Rosa Marlenne Sánchez Sánchez $\mathbb{1}^{\mathrm{b}}$ y Henry \\ Alejandro Silva Marchan $\mathbb{B}^{\mathrm{C}}$
}

-Introducción. -I. Metodología. -II. Resultados y discusión. -Conclusiones. -Referencias.

Primera versión recibida el 10 de noviembre de 2020; versión final aceptada el 29 de diciembre de 2020

\section{Introducción}

El concepto de pandemia deriva del de epidemia, el cual proviene de las palabras griegas epi - por sobre- y demos — pueblo-. En la salud pública, el término se refiere a una enfermedad que afecta a un número de individuos que supera al esperado en una determinada población durante un tiempo específico (Llorca, 2010). Por lo anterior, cuando una enfermedad epidémica se expande a áreas geográficas diferentes, países, e incluso el mundo entero, se le considera como una pandemia, cuyo término según el Diccionario de la Real Academia Española (DRAE) deriva del griego $\pi \alpha \nu \delta \eta \mu i \alpha$ pandèmía que se refiere a "reunión del pueblo" y es definido como "enfermedad epidémica que se extiende a muchos países o que ataca a casi todos los individuos de una localidad o región” (DRAE, 2020).

a Israel Barrutia Barreto: Superintendencia Nacional de Aduanas y Administración Tributaria (SUNAT), Perú. Dirección electrónica: innovasciencia@gmail.com https://orcid.org/0000-0002-5728-0651

b Rosa Marlenne Sánchę Sánchez: investigador y miembro del Grupo Hegemonía, guerras y conflictos, del Instituto de Estudios Políticos de la Universidad de Antioquia, Colombia. Dirección postal: Instituto de Estudios Políticos, oficina 14-209, Calle 70 No. 52-21, Medellín, Colombia. Dirección electrónica: german.valencia@udea.edu.co

https://orcid.org/0000-0002-3434-3293

c Henry Alejandro Silva Marchan: profesor de la Universidad Nacional de Tumbes, Perú. Dirección electrónica: imagenunt@untumbes.edu https://orcid.org/0000-0002-9928-9945

https://doi.org/10.17533/udea.le.n94a344397

\section{(cc) BY-NC-SA}

Este artículo y sus anexos se distribuyen por la revista Lecturas de Economía bajo los términos de la Licencia Creative Commons Atribución-NoComercial-CompartirIgual 4.0. https://creativecommons.org/licenses/by-nc-sa/4.0/ 
Barrutia Barreto, Sánchez Sánchez y Silva Marchan: Consecuencias económicas y sociales...

Durante la historia conocida del hombre se han producido diferentes pandemias, donde las más relevantes han sido la Peste Negra (1348-1400), la Gripe española (1918-1920), el VIH/SIDA, el SARS, la Gripe porcina, la Gripe aviar, Ébola, Zika y, la más reciente, la provocada por el virus SARS-COV-2 conocida como la COVID-19 (Huremović, 2019; Tisdell, 2020). Particularmente, en el siglo XXI se han presentado brotes epidémicos y pandémicos cuyo impacto sobre la economía ha sido importante, sobre todo por sus características de zoonosis, lo cual amerita que la academia, la ciencia y la tecnología se unan para producir respuestas efectivas que reduzcan las consecuencias en el ámbito socioeconómico y de salud pública (Dipaola, 2020; Villamil, 2013).

En el Perú la presencia del virus SARS-COV-2, causante de la COVID-19, se reportó oficialmente el 6 de marzo de 2020 y el día 25 del mismo mes se promulgó el Decreto Supremo N. ${ }^{\circ}$ 094-2020-PCM, el cual estableció "las medidas de aislamiento social destinadas hacia una nueva convivencia social y se prorrogó el Estado de Emergencia Nacional por las graves circunstancias que afectan la vida de la Nación a consecuencia del COV ID-19". Estas medidas sacaron a la luz, no solo las graves deficiencias del sistema de salud sino también cuál debía ser el papel de la industria y la sociedad para hacer frente a la pandemia (Maguiña, 2020).

En ese orden, se ha reportado que el Perú es uno de los países de Latinoamérica con el mayor crecimiento económico desde la década de los años 90, a través de políticas macroeconómicas prudentes y reformas estructurales. Perú pasó de ser un país con tasas de inflación de hasta tres dígitos y bajo crecimiento, a ser una de las economías de mayor crecimiento en América Latina y el Caribe, con cifras de crecimiento promedio del PIB (Producto Interno Bruto) de 5,9\% entre el 2005 y 2015, mientras que la inflación promedio $(2,9 \%$ ) fue una de las más bajas de la región (Carhuaricra y Parra, 2016; Gutiérrez y Pérez, 2018).

La pandemia originada por la COVID-19 y su alto nivel para producir contagios, ha impactado de forma negativa la economía global (Salameh et al. 2020). Es en este sentido, que las acciones de los gobiernos y de la administración pública son fundamentales para producir cambios efectivos 
y sostenibles que promuevan mediante una gestión pública eficiente la recuperación de la economía y la sociedad luego de la pandemia (Acevedo, 2019). En ese orden, el objetivo de esta investigación es indagar sobre las consecuencias económicas y sociales de la inamovilidad humana bajo COVID-19 en el Perú.

\section{Metodología}

El trabajo se basó en una metodología de revisión de fuentes secundarias. Se recopiló información, tanto de portales web donde se han publicado cifras y opiniones de diversos expertos en el área sobre las consecuencias económicas y sociales del aislamiento social en Perú a causa de la COVID19, como de artículos científicos disponibles en la plataforma Science Direct relacionados con el impacto global de la pandemia sobre la economía, y de manera específica los que han tratado el caso de Perú. Las palabras clave de búsqueda utilizadas fueron "Economía", "Impacto socioeconómico", "COVID-19", "Perú", "aislamiento social" e "inamovilidad humana". De igual manera, se recopiló información sobre estadísticas de evolución de la COVID-19 y del comportamiento del mercado económico peruano para poder contrastar la información y llegar a conclusiones.

Las estadísticas sobre indicadores socioeconómicos se obtuvieron del portal oficial del Banco Central de Reserva del Perú (BCRP): PIB y variación respecto al año 2019, puestos de trabajo formal, exportaciones e importaciones totales, índice de precios en Lima Metropolitana e ingreso fueron los indicadores obtenidos. Se estableció un periodo de estudio desde marzo hasta agosto de 2020 debido a los indicadores económicos disponibles. Los datos obtenidos fueron analizados mediante estadística descriptiva y modelos gráficos secuenciales empleando paquetes estadísticos.

\section{Resultados y discusión}

Los casos reportados de COVID-19 han sido monitoreados desde el Ministerio de Salud del Perú, donde se han generado estadísticas detalladas de la evolución de la pandemia. Como caso se tomó el periodo entre marzo 
Barrutia Barreto, Sánchez Sánchez y Silva Marchan: Consecuencias económicas y sociales...

y agosto de 2020. Los casos de contagios reportados se muestran en la Figura 1.

Figura 1. Evolución de la COVID-19 en Perú entre los meses de marzo y agosto 2020

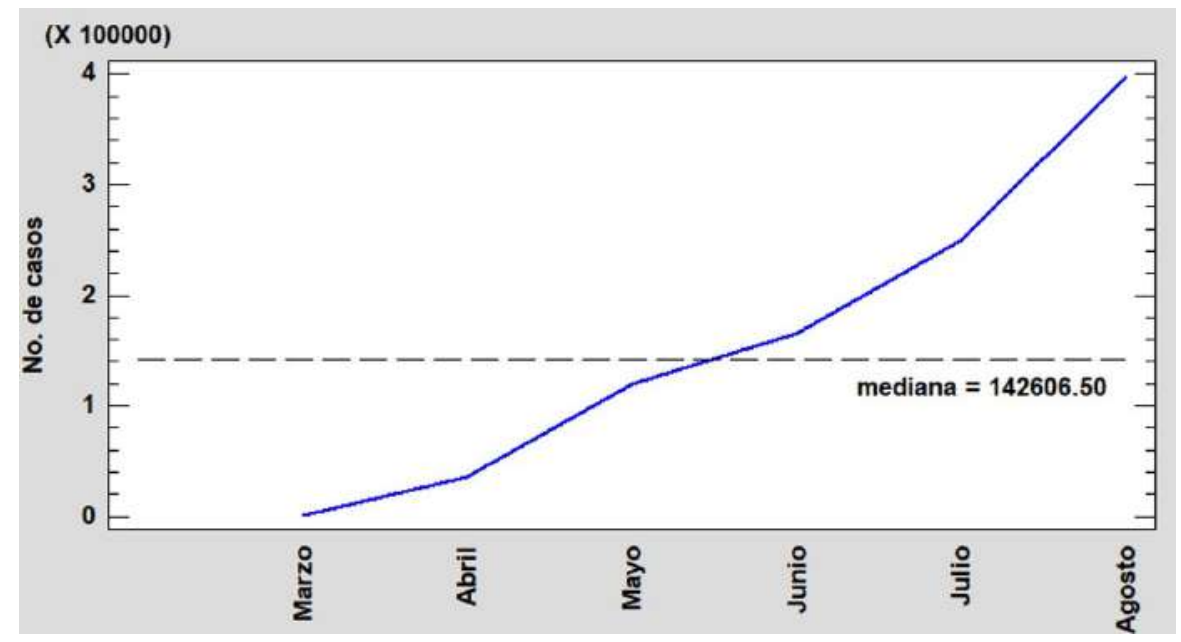

Fuente: propia con datos del MINSALUD (2020).

Se observa que la mediana de los casos durante el periodo de estudio fue de 142606,5 con una rápida evolución desde marzo, donde se reportaron 950 casos, hasta un máximo de 397999 al cierre del mes de agosto. En todo el periodo, la curva de contagios fue en aumento con un incremento fuerte entre julio y agosto, esto a pesar de las medidas de distanciamiento social, aislamiento, toque de queda y otras medidas adoptadas por el gobierno. Una prueba de análisis secuencial temporal indicó que la tendencia creciente de los casos es estadísticamente significativa $(\mathrm{p}<0,05)$, lo que indica un aumento proporcional en el tiempo. Este crecimiento exponencial en el número de contagios reportados se debe, en parte, a la mayor difusión e implementación de pruebas de detección que llevaron a la confirmación más eficiente de los contagios, incluidas a las personas asintomáticas (Aragón y Cruz, 2020, Delgado, 2020).

Durante este periodo la economía se contrajo, sobre todo el PIB, el cual cerró con una diferencia de $-9,8$ \% respecto a agosto de 2019; la mayor caída 
se observó entre los meses de marzo y abril (-39,9\%), coincidiendo con el inicio de las medidas de aislamiento social impuestas por el gobierno, lo que ocasionó incertidumbre en la población y particularmente en los sectores económicos (Figura 2).

Figura 2. Evolución del PIB entre marzo y agosto de 2020 como variación porcentual respecto al mismo periodo del 2019

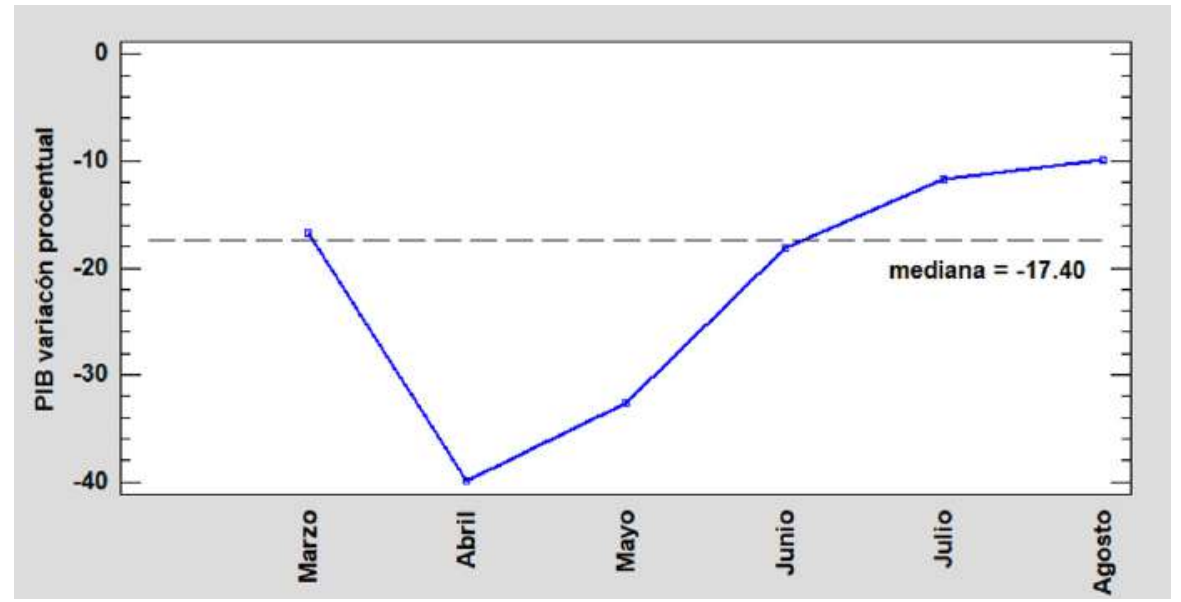

Fuente: elaboración propia con datos del BCRP (2020).

A pesar de la variación negativa media de $-17,4 \%$, es claro que ya había una tendencia que se venía observando desde antes de la aparición del primer contagio por COVID-19, la cual fue acelerada por la enfermedad. Luego de la máxima caída en abril, se fue recuperando, lo que se puede atribuir a la flexibilización de las medidas que - evidentemente- provocaron un aumento en los casos de COVID-19 a pesar de la aparente recuperación económica. El análisis temporal secuencial mostró que no existe un patrón o tendencia definida de este indicador en el periodo de estudio ( $p>0,05)$, lo que es claro al observar la fuerte caída entre marzo y abril.

Según cifras del Ministerio de Economía y Finanzas del Perú (MEF, 2020), la variación del PIB en 2020 — respecto al mismo periodo de 2019 presenta valores positivos hasta el inicio de la pandemia y las medidas de aislamiento social, cerrando en febrero de 2020 en 3,9, y a partir de allí 
Barrutia Barreto, Sánchez Sánchez y Silva Marchan: Consecuencias económicas y sociales...

se observa el comportamiento negativo (lo que es un indicador de que las medidas adoptadas por el gobierno incidieron negativamente). De acuerdo con el Banco Mundial (2020), el PIB del Perú —en términos de porcentaje anual - ha venido decreciendo de forma sostenida desde el año 2010 con una diferencia de $-6,18 \%$. La influencia de la COVID-19 es evidente por acelerar esta tendencia.

Respecto a las exportaciones, estas también se vieron afectadas de forma importante por las medidas de aislamiento social adoptadas entre los meses de marzo y abril, donde se observa una fuerte caída de estas (Figura 3).

Figura 3. Evolución de las exportaciones entre marzo y agosto de 2020

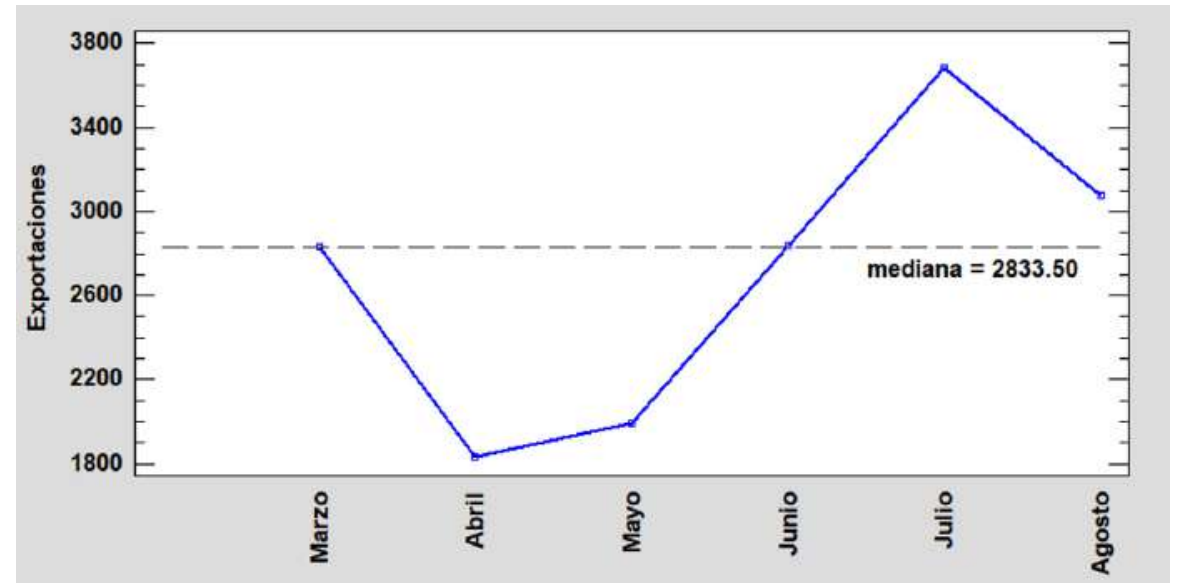

Fuente: elaboración propia con datos del BCRP (2020).

Como se observa, las exportaciones mostraron una mediana de USD 2833,50 millones y no tienen una tendencia definida en el periodo de estudio ( $p>0,05)$. Lo anterior se debe a la caída originada durante el aislamiento social obligatorio. Sin embargo, se produce una recuperación de las exportaciones a partir de la flexibilización de la medida, llegando a recuperar la tendencia observada antes del inicio de la pandemia, ya que en febrero se reportaron USD 3572 millones que fue incluso superado en julio con USD 3572 millones. Según cifras del MEF —y plasmadas en nota de prensa de la agencia EFE América Latina- las exportaciones peruanas sufrieron una caída del 25,8\% 
mensual durante los meses de enero y mayo, debido a una menor demanda internacional, menores precios y, sobre todo, por una disminución de la producción del país ante el impacto de la epidemia de la COVID-19 (EFE, 2020). También se indica que dicha caída en las exportaciones para el mes de mayo fue de $46,2 \%$, aun así, la recuperación observada demuestra que gran parte de la producción se reactivó tras la flexibilización de las medidas de aislamiento social, aun cuando los casos confirmados siguieron en aumento. Lo anterior es evidencia de que, básicamente, se dejó de lado la problemática de salud para reactivar la producción y las exportaciones. Al respecto, el Instituto de Ingeniero de Minas del Perú (2020) reportó que, para el mes de marzo de 2020, la política de aislamiento debido a la COVID-19 impactó negativamente en las exportaciones, las cuales se contabilizaron en USD1689 millones, lo que significó una caída de 17,4\%, con relación a marzo del 2019.

Las importaciones siguieron una tendencia similar a las exportaciones durante el periodo de estudio (Figura 4), con una caída mayor entre los meses de abril a junio.

Figura 4. Evolución de las importaciones entre marzo y agosto de 2020

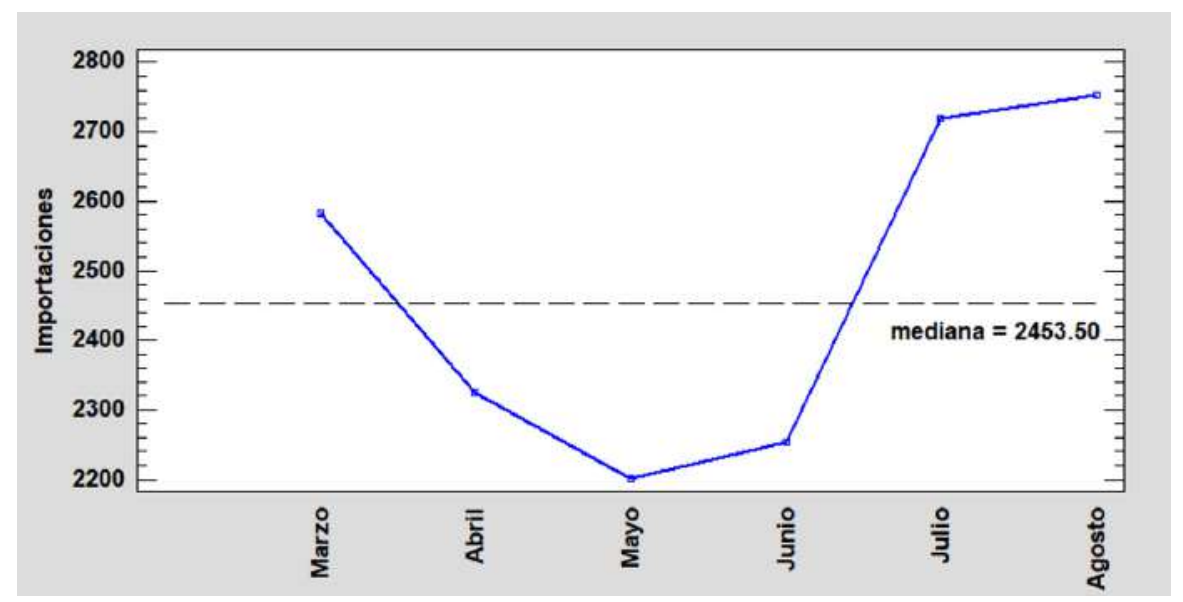

Fuente: elaboración propia con datos del BCRP (2020).

El análisis secuencial temporal de las importaciones mostró una mediana de USD 2453,50 millones con una caída entre los meses de abril y junio, 
Barrutia Barreto, Sánchez Sánchez y Silva Marchan: Consecuencias económicas y sociales...

atribuibles a las medidas tomadas, no solo a nivel nacional sino también mundial, tendientes a controlar la pandemia. Este indicador no siguió en el periodo de estudio una tendencia definida ( $p>0,05)$, y además se observa que se recupera entre los meses de julio y agosto, donde se reactivó el comercio exterior debido a que en muchos países se flexibilizaron las medidas de control. Aunque es claro que el aislamiento social obligatorio influyó en la caída de las importaciones, su tendencia a la baja ya se venía observando entre los meses de enero y febrero, donde se llegó a una diferencia de USD -624,6 millones, representando una caída del 20\%. Según el informe del Instituto Peruano de Economía (2020), la caída de las importaciones debido al aislamiento social afectó a todos los sectores de la economía peruana, con una reducción del 11,7\% respecto al 2019. Lo anterior debido al cierre de puertos y restricciones de intercambio con EE. UU y China (como principales proveedores de productos y servicios), lo que también se destaca en el informe de importaciones presentado por el Centro de Investigación de Economía y Negocios Globales (2020).

Con relación a los puestos de trabajo de la economía formal (Figura 5), también se observa una reducción a partir de las medidas adoptadas por la COVID-19, estando la mayoría de los valores por debajo de la mediana la cual se contabilizó en 3326 miles de puestos.

La caída del empleo formal se observa desde el inicio de las medidas de aislamiento social obligatorio, sin una recuperación visible. A partir de mayo, el indicador se estabiliza con leve tendencia a aumentar, aunque el análisis secuencial mostró que el comportamiento no está definido por una tendencia específica $(p>0,05)$. Es de aclarar que solo se reportan las estadísticas del empleo formal, lo que no refleja la verdadera realidad en un país donde las cifras oficiales del Instituto Nacional de Estadística e Informática del Perú INEI- (2018), indican que el 72,5\% de la población económicamente activa se desempeñan en empleos informales, los cuales fueron más vulnerables ante las medidas de aislamiento social obligatorias. Al respecto, Tenorio (2020) indica que el empleo informal en el Perú es alto y representa el mayor problema del mercado laboral, además reporta que los porcentajes han fluctuado entre $80 \%$ y $70 \%$ en los últimos doce años, sin que se visualice solución para el problema. Según Camero y Pérez (2020) la presencia de la 
COVID-19 en Perú y las medidas de aislamiento social han generado un aumento en la informalidad por una caída de aproximadamente 1,5 millones de puestos de trabajo formal.

Figura 5. Evolución de los puestos de empleo formal entre marzo y agosto de 2020

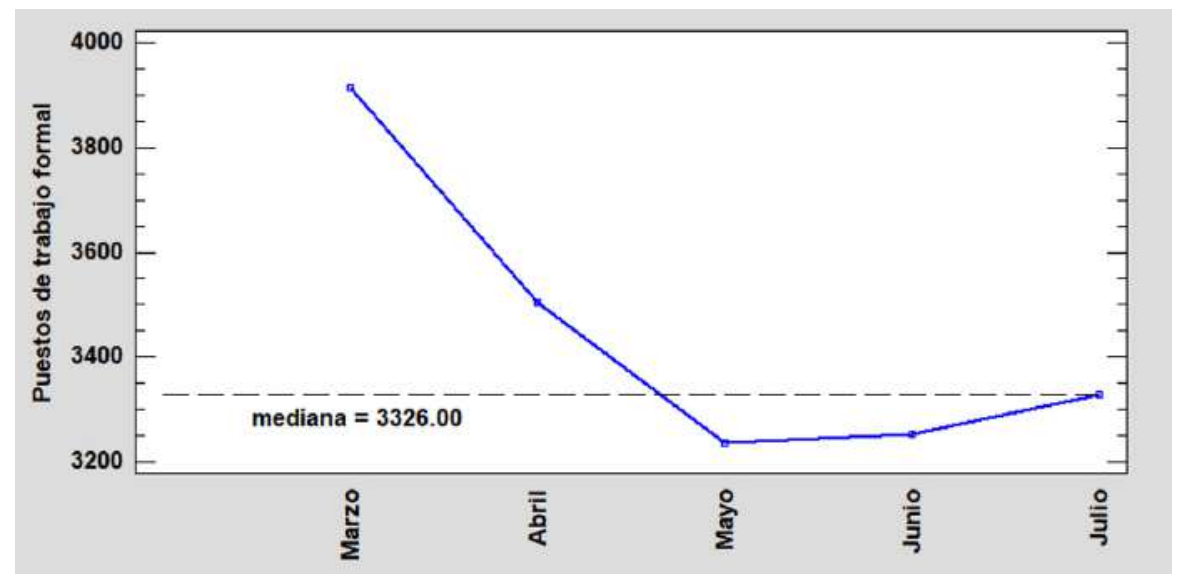

Fuente: elaboración propia con datos del BCRP (2020).

El índice de precios en la ciudad de Lima, aun cuando sufrió fluctuaciones en el periodo de estudio (Figura 6), las mismas no representan una tendencia, según el análisis secuencial $(\mathrm{p}>0,05)$.

El mayor índice de precios se observó en el mes de marzo. Dado que se estaba iniciando con las medidas de aislamiento y existía incertidumbre, los precios aumentaron, lo que llevó al mayor índice del periodo. Este aumento se debe, como lo explica Ayvar (2020) al alza de los precios, sobre todo en algunos productos médicos para evitar el contagio y de otros productos de primera necesidad, debido a la demanda y las compras nerviosas por parte de la población, algo que fue desapareciendo y, posteriormente, se estabilizaron los precios. Lo anterior también se observa en el reporte de índice de precios del INEI (2020) para Lima metropolitana, donde se especifica que el mayor índice lo muestran los productos para cuidado y conservación de la salud, con $0,22 \%$ debido al aumento de las medicinas y los servicios médicos. En el mismo reporte del INEI (2020) se observa que en contraparte, el precio de 
Barrutia Barreto, Sánchez Sánchez y Silva Marchan: Consecuencias económicas y sociales...

los alimentos disminuyó, lo que generó índices negativos y es consistente con lo observado en los meses de junio y agosto con índices de precios negativos.

Figura 6. Evolución del Índice de precios en la ciudad de Lima entre marzo y agosto de 2020

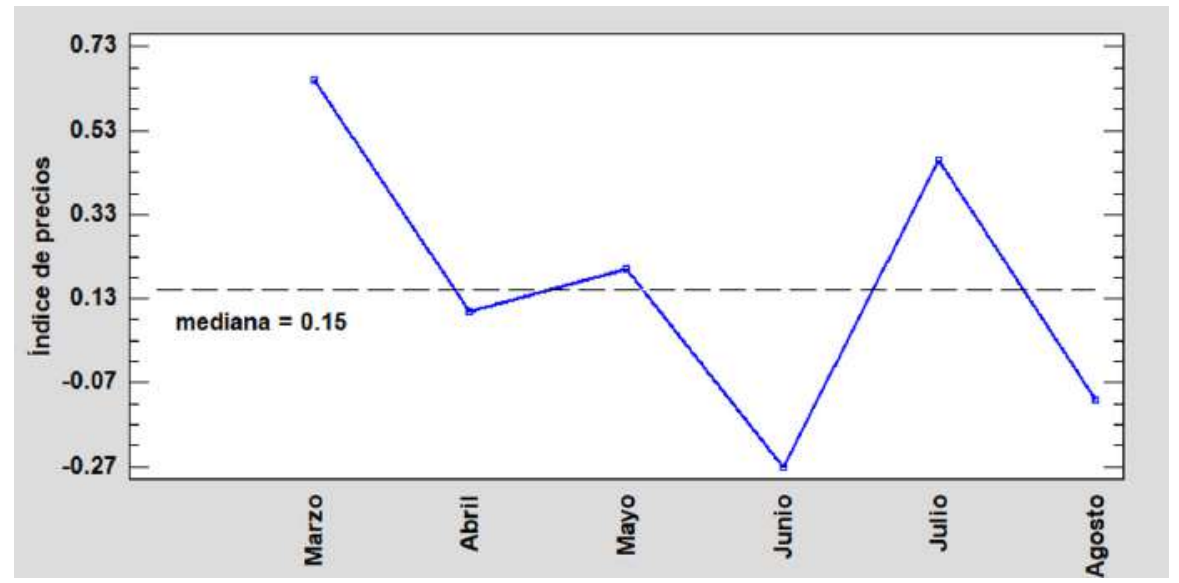

Fuente: elaboración propia con datos del BCRP (2020).

El comportamiento del ingreso promedio se puede observar en la Figura 7, donde los valores fueron reportados hasta el mes de julio por parte del BCRP (2020). Es claro que las medidas gubernamentales para frenar el contagio de la COVID-19 provocaron no solo disminución en el empleo, sino también en los salarios.

Este comportamiento se debe principalmente a que, dadas las medidas de aislamiento, muchas empresas cerraron y otras — cuyos trabajos se podían hacer de forma remota- enviaron a casa a sus colaboradores; pero para poder asumir ese sistema redugeron los sueldos, lo que se sumó al desempleo también impactó el ingreso de las familias. Al irse normalizando la situación con la flexibilización de las medidas, algunas empresas se reactivaron, y por eso se identifica un aumento en el ingreso en el mes de julio, lo que también se vio influenciado por las fiestas patrias, donde los trabajadores formales reciben cierta bonificación. Debido a la pérdida de empleos y la reducción del ingreso como consecuencia de las medidas adoptadas por la COVID-19, 
el Fondo de las Naciones Unidas para la Infancia (UNICEF, 2020) considera que la pobreza monetaria en Perú se ubicaría para el 2020 en 30,3\% lo que representa un aumento de 10,1 puntos porcentuales respecto al año 2019 (aunque este mismo organismo predice una reducción para 2021 a 25,8\% atribuible a que, como se ha venido observando, el sistema económico se ha estado manteniendo a pesar de la pandemia).

Figura 7. Evolución Ingresos entre marzo y agosto de 2020

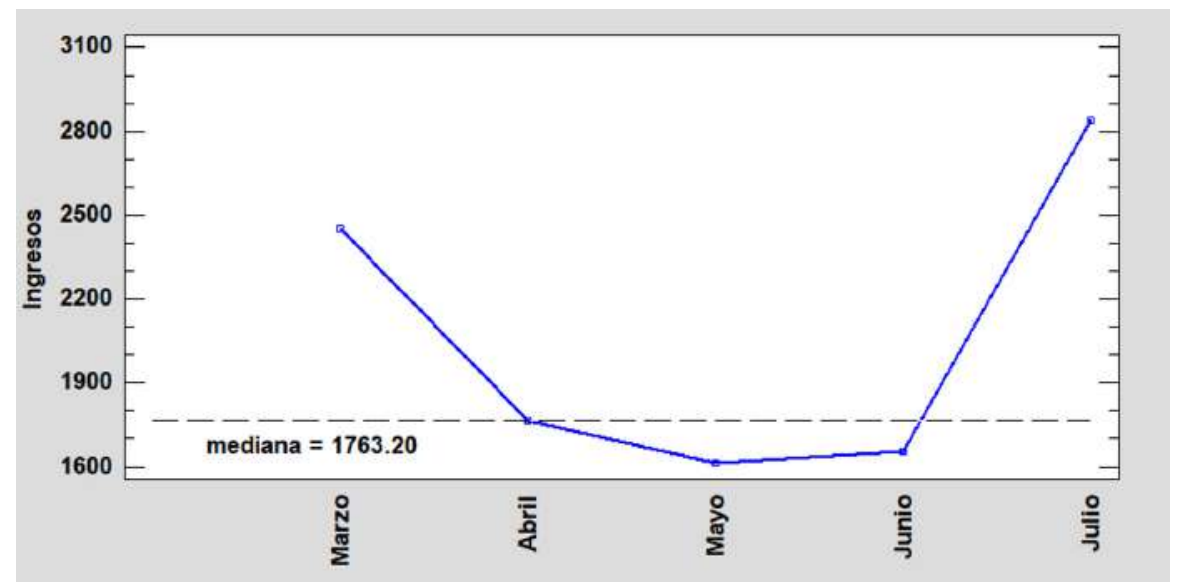

Fuente: elaboración propia con datos del BCRP (2020).

El comportamiento de los indicadores respecto a los casos confirmados de COVID-19 en el periodo marzo-agosto de 2020, se muestran en conjunto de forma gráfica en la Figura 8. Es claro que aun cuando los casos confirmados no dejaron de aumentar, los indicadores tendieron a aumentar hacia el final del periodo.

El mayor impacto se observa al inicio de la presencia de COVID-19 en el país, momento en el que se decretó el aislamiento social obligatorio con restricción de la movilidad y eran pocos los casos reportados. Sin embargo, al flexibilizar las medidas, se observó un incremento en la mayoría de los indicadores sin que los casos disminuyeran. Dicha flexibilización produjo un incremento exponencial de contagios, aun cuando el país se estabilizó o volvió a la tendencia original de sus indicadores socioeconómicos. Con relación a 
Barrutia Barreto, Sánchez Sánchez y Silva Marchan: Consecuencias económicas y sociales...

lo anterior, Kye y Hwang (2020) consideran que las respuestas proactivas por parte de los gobiernos central y locales generan mayor confianza de la población ante la crisis pandémica y eso se asocia con la capacidad para controlar tanto la caída de la economía como el grave problema de salud pública al que se enfrenta.

Figura 8. Indicadores socioeconómicos con relación al número de casos conformados de COVID-19 entre los meses de mayo y agosto del 2010

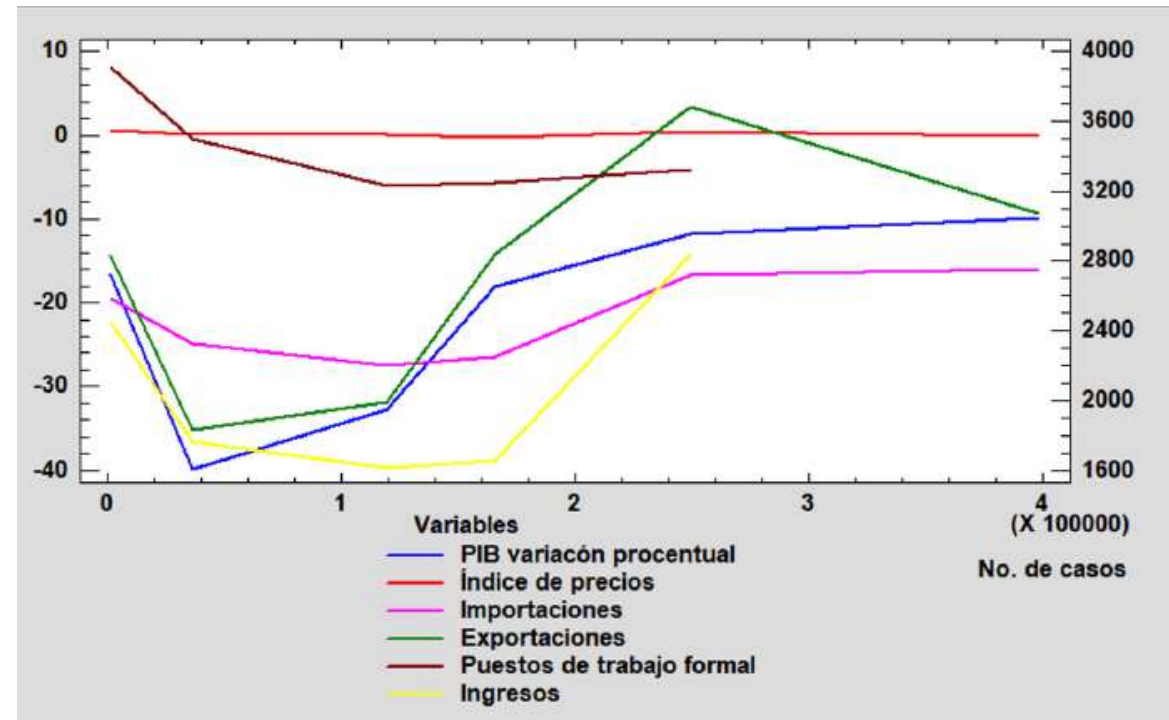

Fuente: elaboración propia a partir de datos del BCRP (2020).

Para Smith-Bingham y Hariharan (2020) la COVID-19 puso en relieve la tendencia de muchos gobiernos a negar o encubrir los casos de contagio y su verdadero impacto en la economía, para evitar sanciones económicas o políticas. En ese mismo orden de ideas Kuckertz et al. (2020) mencionan, respecto a las medidas de aislamiento, que gran parte de la sociedad y de la vida económica ha supuesto una conmoción exógena para muchos agentes económicos, sobre todo para las empresas nuevas de tendencia innovadora que amenazan con su existencia. Así mismo, Vega (2020) considera que las estimaciones que están realizando los organismos multinacionales respecto al impacto de la pandemia sobre la economía mundial, regional y local no están 
considerando las consecuencias ambientales, sociales, culturales, políticas, científicas, tecnológicas y de seguridad y sobre todo en los sistemas de salud.

Por lo anterior es claro que, en el caso peruano, el manejo de la pandemia aun cuando se adoptaron medidas sanitarias que fueron aplicadas a nivel internacional, no ha sido del todo efectivo, ya que con un alto porcentaje de trabajo informal y de personas que viven del día a día como estrategia económica, el confinamiento no es práctico, aunado a esto están las presiones de las industrias para minimizar el impacto en sus finanzas. Ramírez (2020), al referirse a la economía peruana, destaca la posible caída del PIB en 12,7\% y un estimado de 12,4 millones de empleados informales para fines de 2020, lo cual no contribuirá a la disminución de los casos de contagios y muertes, ya que más personas dedicadas al comercio informal aumentarán la circulación del virus y, por ende, los afectados por el mismo, lo que también fue destacado por Marín (2020).

El aumento de la informalidad en el empleo y el consecuente aumento de vendedores ambulantes y puestos de comida en la calle, es un aspecto que se debe tener en cuenta en un país como Perú con su alta tasa de empleos informales, y que puede incidir en el aumento de los casos de contagios y sus efectos adversos. Esto mismo identificó Song (2020) en el caso de China, donde los puestos de comida callejeros son foco de propagación de esta enfermedad y de otras. Lo anterior es consistente con lo observado en el análisis de los indicadores económicos, donde luego de la flexibilización de las medidas se reabrieron muchos comercios y la presencia de la actividad informal en las calles generó una aparente reactivación de la economía, lo que hace que se observe en la calle una gran cantidad de personas, se normalice el transporte público, entre otros. Lo anterior evidentemente influye en que, a pesar de las normas de bioseguridad, el virus circule libremente y se vea el uso de tapabocas y protectores faciales más como una moda impuesta que una protección; y hasta que no se tenga una vacuna, la curva no logrará el ansiado aplanamiento, pues es claro que privan los indicadores económicos y aun el número de fallecidos, para muchos gobiernos representa cifras porcentuales bajas. 
Barrutia Barreto, Sánchez Sánchez y Silva Marchan: Consecuencias económicas y sociales...

\section{Conclusiones}

Es evidente y notorio el impacto de la pandemia generada por la COVID-19 a nivel mundial, no solo en el aspecto de salud sino en la economía de los países, algo de lo que Perú no está exento. Sin embargo, en muchos indicadores económicos se observa que el país ya venía sufriendo retrocesos y fluctuaciones, las cuales fueron aceleradas por las fuertes medidas adoptadas por el gobierno entre los meses de marzo y junio, donde tanto los indicadores económicos como sociales sufrieron una fuerte caída, llevando a la paralización de muchas empresas y despido de personal, lo que a su vez aumentó el desempleo y la mayor presencia de informalidad en un país que de por sí ya tiene arraigado este problema.

A partir de la flexibilización de las medidas de aislamiento social obligatorio, aun cuando se mantuvo la tendencia exponencial creciente de los casos de contagio por COVID-19, se observó una recuperación de los indicadores económicos (PIB, Exportaciones e Importaciones). Sin embargo, el empleo formal no se ha recuperado a los niveles previos al COVID-19, lo que ha generado un aumento en la tasa de informalidad del empleo en el país. Por su parte el ingreso parece haberse recuperado luego de la caída sufrida a raíz de las medidas iniciales, lo que no está acorde con el aumento del desempleo y la informalidad, por lo que este aspecto amerita de un estudio más profundo y más allá de las estadísticas oficiales. Con relación al índice de precios, se observa que al inicio del aislamiento obligatorio los mismos aumentaron al nivel más alto del periodo de estudio, pero se fueron normalizando, regresando a rangos de variación normales que se venían observando antes de las medidas.

El análisis estadístico secuencial temporal indicó que solo los casos de contagio de la COVID-19 tienen una tendencia significativa respecto al tiempo y, como se observó la misma, tiene un comportamiento creciente. El resto de las variables o indicadores estudiados no siguen una tendencia significativa debido a que sus curvas de tendencia se vieron afectadas por las medidas de inmovilidad y aislamiento social que se implementaron al inicio de la presencia de la COVID-19 en Perú, donde los indicadores económicos mostraron una fuerte caída al inicio del periodo y también lo hicieron los 
indicadores sociales; son excepción de los índices de precios, los cuales llegaron al máximo en el mes de marzo. Se debe estudiar a profundidad el verdadero impacto de la pandemia en Perú, ya que en el periodo de estudio (a pesar de que los casos de contagio nunca disminuyeron) los indicadores económicos se estabilizaron a partir de la flexibilización de las medidas de aislamiento social, llegado a presentar la misma tendencia que traían antes de la aparición del primar caso de la enfermedad.

\section{Referencias}

Acevedo, S. I., Barrutia, I., Urquizzo, J. A., \& Venero, R. (2019). La administración pública y sentido de bienestar para el progreso. Religación. Revista de ciencias sociales y humanidades, 4(17), 116-123. http://revista. religacion.com/index.php/religacion/article/view/369

Agencia EFE (2020, 10 de julio). Las exportaciones peruanas cayeron casi $26 \%$ durante cuarentena por COVID-19. https://www.efe.com/efe/america/ec onomia/las-exportaciones-peruanas-cayeron-casi-26-durante-cuarente na-por-covid-19/20000011-4294175

Aragón, J. \& Cruz, M. (2020). Datos y tendencias del Avance del COVID19 en Perú después de 70 días del primer caso reportado y de 60 días de cuarentena [reporte]. Escuela de gobierno y politicas públicas, PUCP. https://escuela.pucp.edu.pe/gobierno/investigacion/reportes-tematicos2/datos-y-tendencias-del-avance-del-covid-19-en-peru-despues-de-70 -dias-del-primer-caso-reportado-y-de-60-dias-de-cuarentena-1/

Ayvar, R. (2020). Los precios y el coronavirus: ¿Intervención o empatía? https:/www.enfoquederecho.com/2020/04/10/los-precios-y-el-coron avirus-intervencion-o-empatia/

Banco Central de Reserva del Perú (2020). Estadísticas. https://www.bcrp.g ob.pe/estadisticas.html

Banco Mundial. (2020). Crecimiento del PIB (\% anual) - Perú. https://datos. bancomundial.org/indicator/NY.GDP.MKTP.KD.ZG?locations=PE 
Barrutia Barreto, Sánchez Sánchez y Silva Marchan: Consecuencias económicas y sociales...

Camero, J. \& Pérez, J. (2020). Perú >Impacto de la COVID-19 en el empleo $y$ los ingresos laborales [nota técnica país]. Organización Internacional del Trabajo. https://www.ilo.org/wcmsp5/groups/public/---americas/---ro -lima/documents/publication/wcms_756474.pdf

Carhuaricra, A. \& Parra, F. (2016). Inversión Extranjera Directa y evolución de las exportaciones: La experiencia peruana en el marco de APEC. Journal of Business 8(1), 72-90. https://doi.org/10.21678/jb.2016.77

Centro de Investigación de Economía y Negocios Globales (2020). Reporte de importaciones. Importaciones peruanas de abril y su impacto por COVID19. http://www.cien.adexperu.org.pe/wp-content/uploads/2020/05/IM PORTACIONES-PERUANAS-DE-ABRIL-Y-SU-IMPACTO-POR -COVID-19-2.pdf

Delgado, D. (2020). La COVID-19 en el Perú: una pequeña tecnocracia enfrentándose a las consecuencias de la desigualdad. Fundación Carolina. https://doi.org/10.33960/AC_26.2020

Diccionario de la Real Academia Española. (2020). Pandemia. Real Academia Española. https://dle.rae.es/pandemia? $\mathrm{m}=$ form

Dipaola, E. (2020). Individualismo y pandemia: consecuencias y riesgos globales. Reflexiones marginales. Número especial 8: coronavirus. https: //revista.reflexionesmarginales.com/individualismo-y-pandemia-consec uencias-y-riesgos-globales/

Fondo de las Naciones Unidas para la Infancia (2020). COVID-19: Impacto en la pobreza y desigualdad en niñas, niños y adolescentes en el Perú. Estimaciones 2020-2021. https://www.unicef.org/peru/media/8866 /file/Impacto\%20COVID19\%20pobreza\%20y\%20desigualdad.pdf

Gutiérrez, A. P. \& Pérez, F. (2018). Choques agregados y sectoriales en la economía peruana. Revista Estudios Económicos, 35, 29-45. https://www. bcrp.gob.pe/docs/Publicaciones/Revista-Estudios-Economicos/35/ree35-perez-gutierrez.pdf

Huremović, D. (2019). Brief history of pandemics (pandemics throughout history). En D. Huremović (Ed.), Psychiatry of Pandemics: A Mental 
Health Response to Infection Outbreak. Springer International Publishing. http://dx.doi.org/10.1007/978-3-030-15346-5_2

Instituto de Ingeniero de Minas del Perú (2020). Efecto del COVID-19: las exportaciones mineras cayeron $17.4 \%$ en marzo. https://iimp.org.pe/pr omocion-minera/efecto-del-covid-19:-las-exportaciones-mineras-cayer on-17.4-en-marzo

Instituto Nacional de Estadística e Informática del Perú (2018). Producción y Empleo Informal en el Perú, Cuenta Satélite de la Economía Informal 20072017. https://www.inei.gob.pe/media/MenuRecursivo/publicaciones_d igitales/Est/Lib1589/libro.pdf

Instituto Nacional de Estadística e Informática del Perú (2020). Precios al consumidor en Lima Metropolitana crecen 0,02\% en octubre. https://ww w.inei.gob.pe/prensa/noticias/precios-al-consumidor-en-lima-metropol itana-crecen-002-en-octubre-12472/

Instituto Peruano de Economía (2020). Informe del Impacto del coronavirus en la economía peruana. https://www.ipe.org.pe/portal/wp-content/uploa ds/2020/03/INFORME-IPE-Impacto-del-coronavirus-en-la-economía -peruana_vf.pdf

Kuckertz, A., Brändle, L., Gaudig, A., Hinderer, S., Morales, C.A., Prochotta, A., Steinbrink, K. M. \& Berger, E. S. C. (2020). Startups in times of crisis-A rapid response to the COVID-19 pandemic. Journal of Business Venturing Insights, 13, e00169. https://doi.org/10.1016/j.jbvi.2020.e0 0169

Kye, B. \& Hwang, S. J. (2020). Social trust in the midst of pandemic crisis: Implications from COVID-19 of South Korea. Research in Social Stratification and Mobility 68, 1005232. https://doi.org/10.1016/j.rssm .2020 .100523

Llorca, J. L. (2010). Introducción al concepto de endemia, epidemia y pandemia [ponencia]. Jornadas sobre prevención de pandemias en las empresas. Institut Valencià de Seguretat i Salut en el Treball, Valencia, Espańa. http://invassat.gva.es/documents/161660384/161741789/Introduc 
Barrutia Barreto, Sánchez Sánchez y Silva Marchan: Consecuencias económicas y sociales...

ci $\%$ C $3 \% \mathrm{~B} 3 \mathrm{n}+\mathrm{al}+$ concepto + de + endemia ++ epidemia $+\mathrm{y}+$ pandemi $\mathrm{a}+2010 /$ cc7afe51-08d0-4008-a865-e21175ad6857

Maguiña, C. (2020). Reflexiones sobre el COVID-19, el Colegio Médico del Perú y la Salud Pública. Acta Médica Peruana 37(1), 8-10. https://doi.org/10.35663/amp.2020.371.929

Marín, T. D. (2020). Editorial: La recuperación de la naturaleza por el aislamiento social debido al COVID-19 ¿Realidad o ficción? Journal of the Selva Andina Research Society 11(2), 60-61. http://ucbconocimiento. ucbcba.edu.bo/index.php/JSARS/article/view/618/557

Ministerio de Economía y Finanzas del Perú (2020). Estadísticas. Política económica y social. https://www.mef.gob.pe/es/component/content/ article $? \mathrm{id}=266$

Presidencia del Consejo de Ministros (2020, 25 de marzo). Decreto Supremo N.o 094-2020-PCM. Que establece las medidas que debe observar la ciudadanía hacia una nueva convivencia social y prorroga el Estado de Emergencia Nacional por las graves circunstancias que afectan la vida de la nación a consecuencia del COVID-19. https://www.gob.pe/instituci on/pcm/normas-legales/584231-094-2020-pcm

Ramírez, M. (2020). COVID-19: ¿Cómo está la economía peruana a 200 días del Estado de Emergencia? https://rpp.pe/economia/economia/covid-19como-esta-la-economia-peruana-a-200-dias-del-estado-de-emergenciareactivacion-economica-fase-4-pbi-bcr-oit-empleo-noticia- 1295793

Salameh, P., Hajj, A., Badro, D. A., Abou, C., Aoun, R. \& Sacre, H. (2020). Mental Health Outcomes of the COVID-19 Pandemic and a Collapsing Economy: Perspectives from a Developing Country. Psychiatry Research, 294, 113520. https://doi.org/10.1016/j.psychres.2020.113520

Smith-Bingham, R. \& Hariharan, K. (2020). El impacto del coronavirus COVID-19 en los negocios. Marsh \& McLennan Insights. https://ww w.marsh.com/co/insights/research/coronavirus-impacto-negocios 
Song, S. (2020). Street Stall Economy in China in the Post-COVID-19 Era: Dilemmas and Regulatory Suggestions. Research in Globalization. https://doi.org/10.1016/j.resglo.2020.100030

Tenorio, D. (2020). El empleo informal en el Perú: una breve caracterización 2007-2018. Pensamiento Crítico, 25(1), 51-76. http://dx.doi.org/10.15 381/pc.v25i1.18477

Tisdell, C. A. (2020). Economic, social and political issues raised by the COVID-19 pandemic. Economic Analysis and Policy 68, 17-28. https://doi.org/10.1016/j.eap.2020.08.002

Vega, E. (2020). Aproximación sociológica para la comprensión de los desacuerdos entre las medidas gubernamentales y la respuesta social frente a la pandemia de Covid-19 en Perú desde un análisis macroestructural. https://www.clac so.org/los-desacuerdos-entre-las-medidas-gubernamentales-y-la-respue sta-social-frente-a-la-pandemia-de-covid-19-en-peru/

Villamil, L. C. (2013). Epidemias y pandemias: una realidad para el siglo XXI. Un mundo y una salud. Revista Lasallista de Investigación 10(1), 7-8. http://repository.lasallista.edu.co:8080/ojs/index.php/rldi/article/ view/418/191

Cómo citar / How to cite this item:

Barrutia-Barreto, I., Sánchez-Sánchez, R. M. \& Silva-Marchan, H. A. (2021). Consecuencias económicas y sociales de la inamovilidad humana bajo COVID-19 caso de estudio Perú. Lecturas de Economía, 94, 285-303.

https://doi.org/10.17533/udea.le.n94a344397 